\title{
Autologous Blood-Derived Factors in Regulation of Inflammation and Enhancement of Healing, New Strategies and Perspectives
}

\section{Joanna Wessely-Szponder*}

Department of Pathophysiology,Chair of Preclinical Veterinary Sciences, Faculty of Veterinary Medicine, Akademicka, Lublin, Poland

"Corresponding author: Joanna Wessely-Szponder, Department of Pathophysiology,Chair of Preclinical Veterinary Sciences, Faculty of Veterinary Medicine, Akademicka, Lublin, Poland. Tel: +48814456774; Email:joanna.wessely@up.lublin.pl

Citation: Wessely-Szponder J (2017) Autologous Blood-Derived Factors in Regulation of Inflammation and Enhancement of Healing, New Strategies and Perspectives. Arch Vet Sci Technol: AVST-126. DOI:10.29011/AVST-126/100026

Received Date: : 20 July, 2017; Accepted Date: 29 July, 2017; Published Date: 10 August, 2017

\section{Editorial}

Recently, so called "Autologous Biomaterials", as naturally occurring substances are considered to have a potential for enhance the bone and soft tissue healing and help fight infection [1]. From these biomaterials the Platelet-Rich Plasma (PRP) - a preparation of concentrated autologous platelets containing many bioactive factorsis widely used in clinical practice, whereas antimicrobial neutrophil extract (AMP) is taken into account as a new autologous preparation for enhance of tissue repair. PRP was previously used in animals and humans in clinical therapies of many disorders in dentistry, neurosurgery, cardiothoracic, and maxillofacial surgery, in orthopedics and traumatology [2]. Neutrophilderived antimicrobial peptides, in turn, were applied in treatment of osteomyelitis. However, to date combination of both preparations has not been used in clinical practice. We assumed that both autologous preparations used together could be applied locally in surgical and orthopedic conditions to enhance healing. Therefore, the research preceding clinical local use of both these preparations in veterinary practice has been started. For this purpose, PRP rich in growth factors and with restricted amount of WBC has been prepared. Next, we evaluated the method for preparation of AMP neutrophil extract containing natural antimicrobial peptides.Moreover, in order to achieve a better understanding of the contribution of inflammatory cells in the healing process the influence of AMP extract on the activity of neutrophils and macrophages in vitrowas also assessed [3].

Cationic antimicrobial peptides are very common in domesticated animals and vary considerably in their composition, structure and distribution among species. These peptides, especially their influence on inflammatory cells and regulation of inflammation as well as repair process in multiple tissues are currently of great interest. Since antimicrobial peptides and proteins can be offered as possible replacement of antibiotics which lost their effec- tiveness due to microbial resistance, the alternative means are of great importance. Among antimicrobial peptides cathelicidins are effector molecules of innate immunity with diverse functions, including antimicrobial activity, modulation of wound repair and inflammation by influence on inflammatory cells. Within these cells, macrophages initially contribute to the elimination of pathogens and the elicitation of an inflammatory reaction. When the infection is resolved their function may be directed towards resolution of inflammation and repair process. Macrophages can thus exhibit pro and anti-inflammatory properties, and as reported previously antimicrobial peptides i.e. defensins and cathelicidins may participate in this regulation [4].

In the light of fact that the major challenge for many analyses of physiologic fluids is to identify optimal procedures for sample preparation, in both cases reliable qualitative method to detection the components of these bioactive preparations is necessary. In many cases only very low amounts of biological specimen are available for the experiment. MALDI-TOF MS is the method that provides rapid determination of molecular masses and the heterogeneity of small amounts of peptides and proteins. The limits of detection for MALDI-TOF MS depend on many variables that may change detection sensitivity. However, for small peptides, under optimal conditions, limits of detection can extend to $1 \mathrm{nmol} / 1$ solution in $1 \mu \mathrm{l}$. Another advance of MALDI-TOF MS is that peptides smaller than $7000 \mathrm{Da}$ are not detected by standardtechniques of 2-dimensional electrophoresis because they are below the limits of size resolution, and small components may not be fixed in gel and produce lower staining intensity per mole of peptide. Most electrophoretic and chromatographic techniques preferentially detect large molecules,because absorbance or intensity of staining per molar equivalent of protein generally increases in proportion to size. Consequently, MALDI-TOF MS has served as a useful tool to identification and characterization of small peptides as well as to open up peptidomic analysis [5]. 


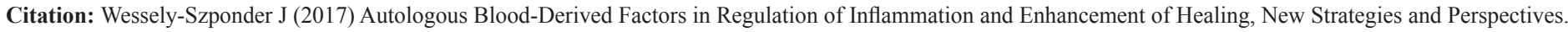
Arch Vet Sci Technol: AVST-126.

There are many protocols to obtain PRP, without one standardized method. In case of AMP extract, in turn, we described the method to obtain this preparation. Due to a small volume of the obtained blood the MALDI TOF analysis is sufficient for preliminary qualitative detection of some bioactive factors during the standardization of method of preparation of PRP and AMP extract in many species. The modified method permits direct analysis of biological samples without previous preparation steps that involve significant time, expense, and potential losses of selected components. Using MALDI TOF for qualitative determination of bioactive components it is possible to asses these factors even if ELISA tests are unreliable because of species differences [6].

Our method permits to evaluate two autologous biomaterials for topical application; PRP rich in growth factors and AMP neutrophil extract which comprisesantimicrobial peptides; cathelicidins and defensins with a broad spectrum of antimicrobial activity. These blood-derived products can be used to enhance healing and ensure antimicrobial activity without side effects induced by intact neutrophils. Therefore, the crude neutrophil extract could be used for improvement of antimicrobial activities of PRP without the enlargement of the WBC number. The addition of AMP extract into cultures of neutrophils and macrophages revealed that this extract decreased superoxide anion in culture of macrophages and inhibited degranulation and respiratory burst in neutrophils, thus acts by decrease of proinflammatory effect on studied subpopulations of white blood cells [3].

Detailed study concerning the process of macrophages activation and its regulation during tissue repair should be useful in the introduction of new therapies based on manipulation ofmacrophages function for enhance healing [7].The first part of these studies concerning the role of antimicrobial peptides in activation of monocyte-derived macrophages during implantation of titanium tibial implants has been just finished [8].Response of activated macrophages to stimulation by AMP extract may have therapeutic role in enhancement of tissue repair and remodeling, especially, for patients with impaired healing and regenerative capacity.The modification of phenotype of macrophages and their products for therapeutic purpose create new strategy for safe and effectivemethod of improve healing and repair with reduced risk of complications.

\section{References}

1. Jameson C (2007) Autologous platelet concentrates for the production of platelet gel. Lab medicine 38: 39-42.

2. Amable PR, Carias RBV, Teixeira M, Pacheco IC, do Amaral RC (2013) Platelet-rich plasma preparation for regenerative medicine: optimization and quantification of cytokines and growth factors. Stem Cell Res. \& Ther 4: 67

3. Szponder T, Wessely-Szponder J, Smolira A (2017)Evaluation of platelet-rich plasma and neutrophil antimicrobial extract as two autologous blood-derived agents. Tissue Eng. Regen. Med3: 287-296.

4. Does A, Beekhuizen H, Ravensbergen B, Vos T, Ottenhoff TH (2010) LL-37 direct macrophage differentiation toward macrophages with proinflammatory signature. J. Immunol 185: 1442-1449.

5. Hortin G (2006) The MALDI-TOF Mass Spectrometric view of the plasma proteome and peptidome. Clin. Chem52:1223-1237.

6. Smolira A, Wessely-Szponder J (2015) Importance of the Matrix and the Matrix/Sample Ratio in MALDI-TOF-MS Analysis of Cathelicidins Obtained from Porcine Neutrophils Applied Biochemistry and Biotechnology Part A: Enzyme Engineering and Biotechnology 175: 20502065.

7. Novak M, Koh T (2013) Phenotypic transitions of macrophages orchestrate tissue repair. Am. J. Pathol 183: 1352-1363.

8. Wessely-Szponder J, Szponder T, Bobowiec R (2017) Different activation of monocyte-derived macrophages by antimicrobial peptides at a titanium tibial implantation in rabbits. Research in Veterinary Science 115: 201-210. 